\title{
Effect of interchain separation on the photoinduced absorption spectra of polycarbazolyldiacetylenes
}

\author{
D. Comoretto, I. Moggio, C. Dell'Erba, C. Cuniberti, G. F. Musso, and G. Dellepiane \\ Dipartimento di Chimica e Chimica Industriale, Università degli Studi di Genova, via Dodecaneso 31, I-16146 Genova, Italy \\ L. Rossi and M. E. Giardini \\ INFM-Dipartimento di Fisica “A. Volta,', Università degli Studi di Pavia, via Bassi 6, I-27100 Pavia, Italy \\ A. Borghesi \\ INFM-Dipartimento di Fisica, Università degli Studi di Modena, via Campi 213/a, I-41100 Modena, Italy
}

(Received 28 May 1996; revised manuscript received 8 August 1996)

\begin{abstract}
The photoinduced absorption spectra of a novel polycarbazolyldiacetylene with long aliphatic chains on the carbazolyl side groups are measured and compared with those of the unsubstituted polyDCHD. The two polymers in the blue form exhibit very similar electronic absorption spectra and Raman frequencies. This fact indicates that the conjugation length of the polydiacetylene backbone is not too affected by the long substituents. In contrast, the near steady-state photoinduced absorption spectra show that different photogeneration mechanisms are involved in the two polymers. This result can be ascribed to the role played by the interchain distance in the dynamics of the relaxation processes in polydiacetylenes. [S0163-1829(96)07747-8]
\end{abstract}

During the last decade the study of polydiacetylenes (PDA's) and in particular of their electronic excited states has been one of renewed increasing interest. This happened both because new information has become available from pump-and-probe and steady-state experiments ${ }^{1}$ and because new oriented forms have been prepared that have provided additional data on the polarization dependence of their photophysical properties. ${ }^{2}$ Among PDA's, polyDCHD (poly[1, 6-bis(N-carbazolyl)-2,4-hexadiyne]) has been of particular interest for the very high value measured for its third-order electrical susceptibility. ${ }^{3,4}$ However, polyDCHD is insoluble so that the exploitation of its peculiar properties still remains at a potential level. In order to obtain a soluble polymer with electronic properties similar to those of polyDCHD, we have prepared a new polyDCHD (polyDCHD-S, i.e., poly[1, 6-bis(3,6-didodecyl-N-carbazolyl)-2,4-hexadiyne]) that is soluble in the most common organic solvents, although its solubility is not as good as that of the well known PDA-4BCMU. ${ }^{5}$ PolyDCHD-S exhibits two different forms, the blue one (with longer conjugation length) and the red one. The blue form is obtained when the monomer is polymerized in $\mathrm{KBr}$ pellets ${ }^{6}$ or when the polymerization is carried out on the cast monomer mechanically aligned along a direction on a hot substrate. The red form is observed in solution and in cast films. A mixture of both blue and red forms is obtained by mass polymerizing the monomer. It is important to note that for polyDCHD-S it is possible to obtain oriented samples in both blue and red forms by mechanical alignment. Oriented samples are, however, not homogeneous on large scale due to the rather poor solubility of the polymer.

In a previous work, we have reported a spectroscopic study of the blue form of polyDCHD-S. ${ }^{6}$ The analysis of all the relevant data indicates that the effective conjugation length of polyDCHD-S is similar to that of polyDCHD, and that the interchain separation is greater in polyDCHD-S with respect to polyDCHD. The purpose of this paper is to report the photoinduced absorption spectra of polyDCHD-S in the blue form and to compare them with those already obtained for polyDCHD (Refs. 7 and 8) in order to clarify the influence of the interchain separation on the nature and dynamics of the long-lived photogenerated states. These photoexcitations turn out to be strongly dependent on the interchain coupling among the $\pi$-conjugated chains.

The electronic absorption and photoinduced absorption (PA) spectra have been recorded by using the techniques described in previous works. ${ }^{7,8}$

Figure 1 shows the absorption and photoluminescence (PL) spectra of polyDCHD-S at $T=77 \mathrm{~K}$ to be compared with those of polyDCHD. ${ }^{6}$ The introduction of long alkyl chains on the carbazolyl side groups does not essentially alter the conjugation length of the polydiacetylene backbone as can be deduced from the absorption spectra of both polymers which exhibit singlet excitonic transitions (at $1.88 \mathrm{eV}$ for polyDCHD-S and at $1.86 \mathrm{eV}$ for polyDCHD) followed by a vibrational progression (less pronounced in polyDCHD$\mathrm{S})$. This behavior was interpreted in terms of a more ordered structure of the soluble polymer. ${ }^{6}$ The sharpening of the excitonic peak on going from polyDCHD to polyDCHD-S was considered an additional piece of evidence. The PL spectrum of polyDCHD-S shows only a peak at $1.78 \mathrm{eV}$, the second weak feature at $1.9 \mathrm{eV}$ resulting from the self-absorption of the polymer due to the overlap of the emission and the absorption spectra in this region. This is in contrast with the rich features exhibited below $1.75 \mathrm{eV}$ by the luminescence spectrum of polyDCHD due to a strong vibrationalelectronic coupling. ${ }^{7}$

Figure 2 reports the PA spectra of polyDCHD-S. The inphase detection spectrum shows only an intense PA peak at $1.45 \mathrm{eV}$ with a shoulder at about $1.35 \mathrm{eV}$. The expected 


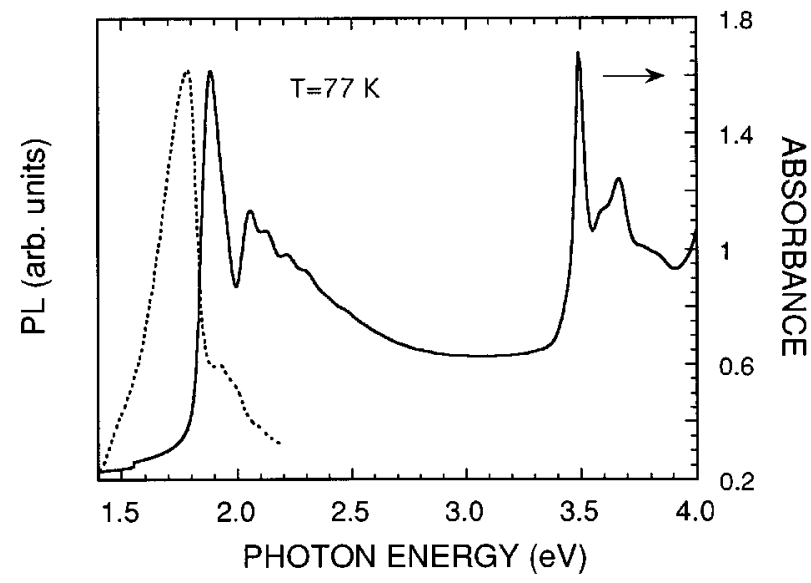

FIG. 1. Absorbance (full line) and PL (dashed line) spectra of polyDCHD-S.

bleaching at $\sim 1.8 \mathrm{eV}$ is not evident due to the PL signal present in this region. In the out-of-phase detection, the PA signal is strongly reduced and very weak features become evident at $0.76 \mathrm{eV}$ and at $0.88 \mathrm{eV}$ (see the inset of Fig. 2). Note that the bleaching at $\sim 1.8 \mathrm{eV}$ is the most prominent structure in this spectrum. The different phase dependence of the $1.45 \mathrm{eV}$ peak with respect to those at 0.88 and $0.76 \mathrm{eV}$ clearly shows that nonlinear excitations with different lifetimes are photogenerated in this polymer. On the contrary, the in-phase PA spectrum of polyDCHD exhibits three photoinduced peaks of about the same intensity. ${ }^{7}$ The higherenergy peak at $1.26 \mathrm{eV}$ is absent in the out-of-phase detection, while the 0.81 - and $0.96 \mathrm{eV}$ peaks remain quite evident. Moreover, in this polymer, a series of infrared active vibrational (IRAV) bands are observed as Fano windows due to the overlap of the IRAV modes with a low-energy electronic band at $\sim 0.1 \mathrm{eV}{ }^{8}$ This detailed study has pointed out that the 0.81 - and $\sim 0.1 \mathrm{eV}$ bands are associated with bipolaronic (BP) states (the $0.96 \mathrm{eV}$ signal is the vibronic replica of the higher-energy bipolaronic level). In the same study the 1.26

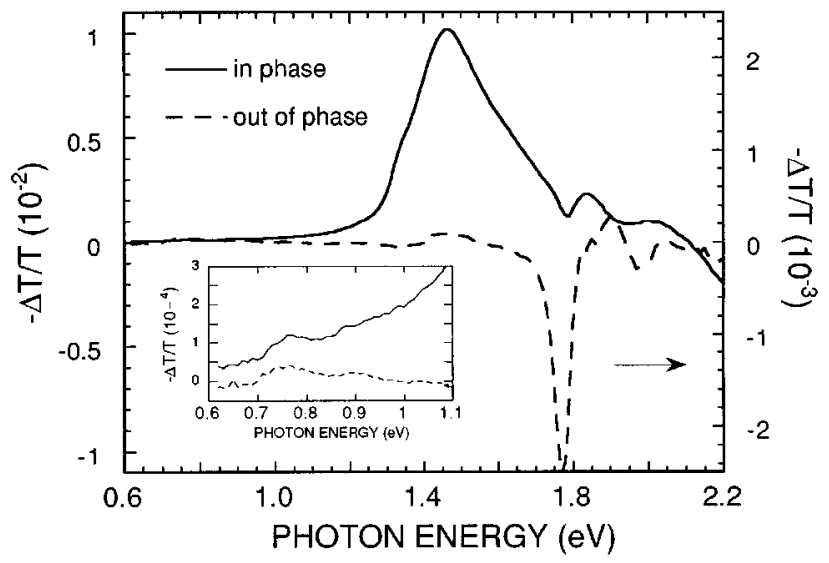

FIG. 2. Photoinduced absorption (PA) spectra of polyDCHD-S $\left(T=77 \mathrm{~K}, \lambda_{\text {exc }}=488 \mathrm{~nm}, P=200 \mathrm{~mW}\right.$, chopper frequency $=20$ $\mathrm{Hz}$ ). Inset: PA spectra of polyDCHD-S in the $0.6-1.1-\mathrm{eV}$ spectral region.

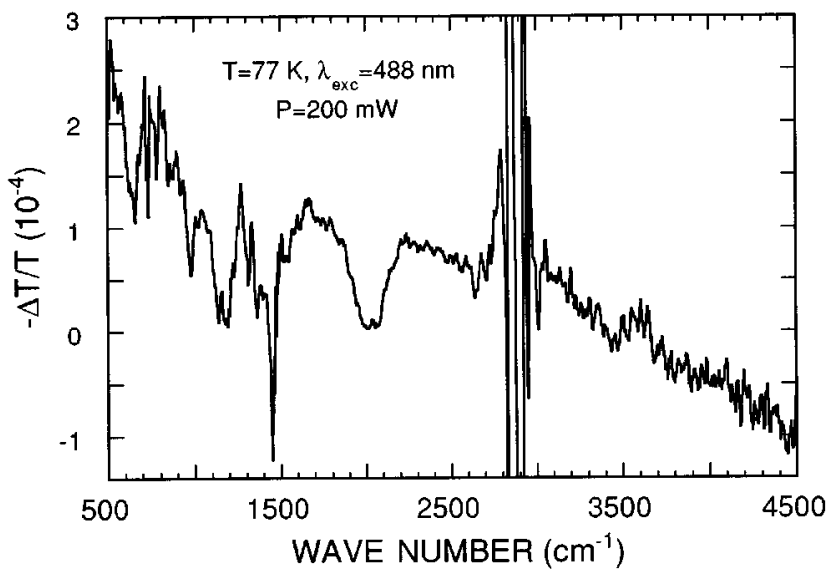

FIG. 3. PA spectra of polyDCHD-S in the MIR spectral region from $500-4500 \mathrm{~cm}^{-1}$.

eV signal, which is clearly originated from nonlinear excitations of different nature, has been assigned to a triplet-totriplet transition. This assignment has been confirmed by the study of the pump polarization dependence of the photoinduced signals in highly oriented samples where it was shown that BP's are preferentially generated relative to triplets by pumping radiation polarized perpendicularly to the chain direction that is by activation of the interchain hopping mechanisms. ${ }^{2}$ The assignment to BP's of the two subgap PA bands which behave as described in Ref. 7, commonly accepted in the literature, has been recently questioned by Shimoi, Abe, and Harigaya. ${ }^{9}$ On the basis of a single chain theoretical model, these authors conclude that the inclusion of electron-electron interaction does not modify the number of predicted subgap bands which is two for polarons and only one for BP's. Nevertheless, the question still appears open and for this reason we have decided to maintain the original assignment for the moment. On the other hand, the precise nature of the charged species is not of particular relevance for the present paper, whose main objective is rather to compare the long-time PA response in the two polymers. We thus believe that the weak $0.76-$ and $0.88 \mathrm{eV}$ peaks in polyDCHS-S can be assigned to BP since their behavior upon changing the phase detection is similar to that of polyDCHD. Moreover, the similarity of the phase dependence of the high-energy peak in the two polymers suggests its assignment to the same type of transition. Notice, however, the very strong $1.45 \mathrm{eV}$ signal $\left(-\Delta T / T \sim 10^{-2}\right)$ in polyDCHD-S against the much weaker $1.26 \mathrm{eV}$ peak $\left(-\Delta T / T \sim 10^{-4}\right)$ in polyDCHD. Notice further that, even though in polyDCHD-S the BP's are present, their photogeneration is strongly reduced relative to that of the nonlinear excitations responsible for the $1.45 \mathrm{eV}$ peak. The presence of charged states in polyDCHD-S is also confirmed by the photoinduced absorption spectrum in the medium infrared reported in Fig. 3 which can be compared with that of polyDCHD. ${ }^{8}$ Except for the thermal modulation of the $\mathrm{CH}$ stretching $\left(\sim 2900 \mathrm{~cm}^{-1}\right)$ and $\mathrm{HCH}$ bending $(\sim 1450$ $\mathrm{cm}^{-1}$ ) modes, polyDCHD-S exhibits features similar, although weaker, to those of polyDCHD. The complex line shapes of the IRAV modes in these polymers do not allow 


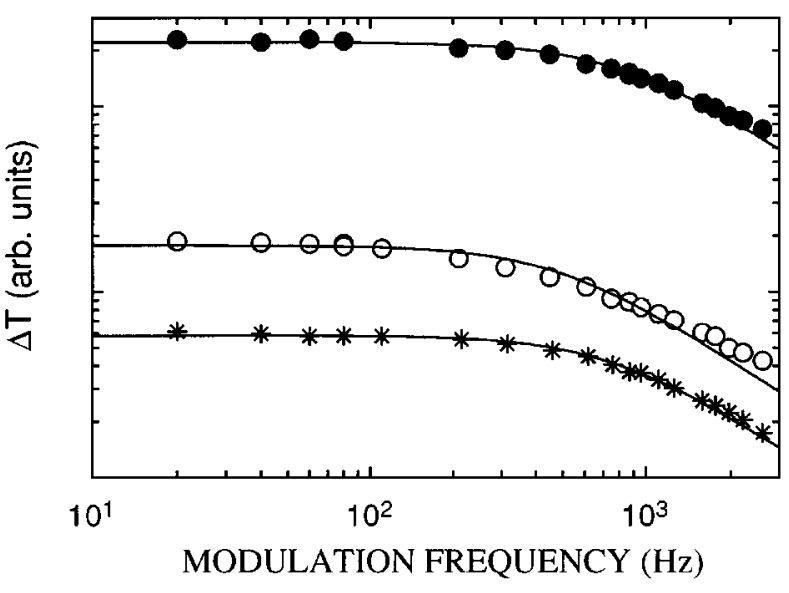

FIG. 4. Modulation frequency dependence of the $\Delta T$ signal at $1.45 \mathrm{eV}$ for polyDCHD-S. $T=77 \mathrm{~K}, P=200 \mathrm{~mW}$ (closed circles); $T=77 \mathrm{~K}, P=70 \mathrm{~mW}$ (open circles); $T=20 \mathrm{~K}, P=70 \mathrm{~mW}$ (stars). The lines are the best-fit results according to a monomolecular decay law.

the differences in their frequency values to be determined. On the other hand, the vibrational frequencies of the blue form of polyDCHD-S have been found to be very close to those of polyDCHD. ${ }^{6}$

The $1.45 \mathrm{eV}$ peak may be assigned to a triplet-to-triplet transition on the basis of its dependence on the laser power $(\mathrm{P})$, modulation frequency, and temperature. A further demonstration of the assignment of this peak to neutral excitations comes from the absence of strong oscillations near the band edge which are instead observed in polyDCHD where charged states are dominant. ${ }^{7}$ The PA signal at $1.45 \mathrm{eV} \mathrm{de}-$ pends on pump intensity as $I^{0.7}$ in the range $20-300 \mathrm{~mW}$, thus indicating that both monomolecular and bimolecular recombination kinetics take place. The frequency dependence of the PA signal at $1.45 \mathrm{eV}$ is shown in Fig. 4. At $T=77 \mathrm{~K}$ and for both $P=70$ and $200 \mathrm{~mW}$ the PA signal is constant for frequencies up to approximately $200 \mathrm{~Hz}$ and begins to roll off for higher frequencies. Since the knee positions do not change with the laser power, we infer that the monomolecular decay is dominant. In the case of bimolecular recombination kinetics, the knee position should change according to the square root of the intensity of the laser power. ${ }^{7,10}$ The frequency dependence at $20 \mathrm{~K}$ shows a knee at a chopper frequency lower than that at $77 \mathrm{~K}$. By nonlinear least-square fittings of the curves, the lifetimes values of $2 \times 10^{-4} \mathrm{~s}$ at $T=77 \mathrm{~K}$ and of $3 \times 10^{-4} \mathrm{~s}$ at $T=20 \mathrm{~K}$ have been calculated. The very small increase of the lifetime with decreasing temperature indicates a somewhat more efficient trapping of the excitations upon lowering the temperature. Consequently, no appreciable change in band intensity with the temperature is expected for the $1.45 \mathrm{eV}$ signal, in agreement with the observed PA spectra shown in Fig. 5. A very similar behavior is observed for the $1.35 \mathrm{eV}$ shoulder. On the other hand, the lifetime of the excitation responsible for the $1.26 \mathrm{eV}$ peak in polyDCHD has been found to be less than $10^{-4} \mathrm{~s}$ at $77 \mathrm{~K}$ and $2.3 \times 10^{-4} \mathrm{~s}$ at $20 \mathrm{~K}$. This lifetime change with the temperature accounts for the threefold increase of the band intensity upon lowering the temperature from 77 to $20 \mathrm{~K}$. Life-

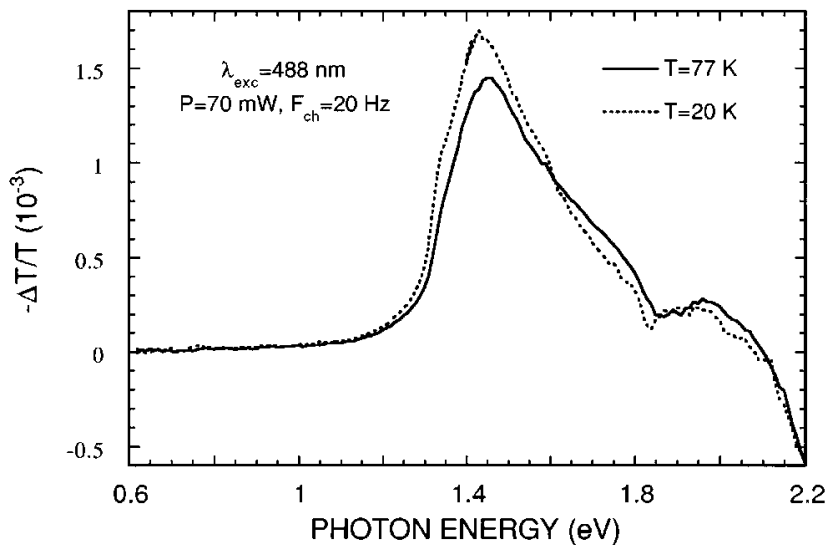

FIG. 5. PA spectra of polyDCHD-S at two different temperatures.

time values of the same order of magnitude have been reported for oligothiophenes ${ }^{11}$ and PDA-4BCMU. ${ }^{12}$ The PL signal dependence on chopping frequency was found to be constant over the full range considered $(20-2000 \mathrm{~Hz})$, indicating that the PL lifetime is much lower than $10^{-4} \mathrm{~s}$.

In conclusion, the PA spectra of polyDCHD-S reveal the presence of a very intense band at $1.45 \mathrm{eV}$, tentatively assigned to triplet excitons, in addition to weak features attributed to bipolarons. In this polymer the photogeneration of long-lived BP states is reduced in favor of long-lived triplet excitons in such a way that only our previous knowledge of the PA spectrum of polyDCHD allowed us to detect the doubly-charged excitations. This result can be explained in terms of the different interchain separation of the two polymers. In fact, the formation of BP's is favored by strong interchain interactions. The same explanation for the different origin of the PA peaks observed in several PDA's has been reported. ${ }^{7}$ Here, it is worth underlining that the detection of a strong single PA band around $1.2-1.5 \mathrm{eV}$ in PDA's, usually associated with triplet excitons, ${ }^{12-14}$ does not always mean that BP's are absent, but that their concentration is much lower than that of triplet excitons. On the other hand, only BP's have been observed in PDA's with short substituents as polyCPDO (Ref. 15), polyCPDA (Refs. 10 and 16), and PDA-1OH. ${ }^{17}$ The absence of the near-steady state PA peak associated with triplet excitons in these systems may be attributed either to a very low efficiency of triplet formation or to a very short triplet lifetime. The picture emerging from this discussion suggests the need for a critical revision of the photoexcitations in PDA's in different aggregation states, time regimes, and spectral regions. We are now left with the dilemma concerning the fact that the PA peaks associated with triplet excitons of polyDCHD-S and polyDCHD (which exhibit very similar singlet exciton transitions, Raman frequencies, and BP transitions) are quite different in transition energy, band shape, and temperature dependence. In particular, the $1.26-\mathrm{eV}$ peak of polyDCHD is sharp and so strongly dependent on the temperature that it becomes the most intense peak only below $80 \mathrm{~K}$. This could be indicative of the presence of almost-free triplet excitons, which become 
trapped only at low temperature. In contrast, the $1.45 \mathrm{eV}$ peak of polyDCHD-S is rather broad and does not practically change with temperature. We believe that this is evidence of a broad energy band associated with trapped triplet excitons deeper in the gap than the triplet level of polyDCHD. The reason for this behavior is rather obscure, as all the experimental results suggest that a better intramolecular order was achieved for polyDCHD-S.
${ }^{1}$ K. Ichimura, M. Yoshizawa, H. Matsuda, S. Okada, M. M. Ohsugi, H. Nakanishi, and T. Kobayashi, J. Chem. Phys. 99, 7404 (1993); M. Yoshizawa, Y. Hattori, and T. Kobayashi, Phys. Rev. B 47, 3882 (1993).

${ }^{2}$ D. Comoretto, G. Dellepiane, C. Cuniberti, L. Rossi, A. Borghesi, and J. Le Moigne, Phys. Rev. B 53, 15653 (1996); D. Comoretto, G. Dellepiane, C. Cuniberti, G. F. Musso, L. Rossi, A. Borghesi, and J. Le Moigne, Synth. Met. 76, 27 (1996).

${ }^{3}$ M. Nisoli, V. Pruneri, V. Magni, S. De Silvestri, G. Dellepiane, D. Comoretto, C. Cuniberti, and J. Le Moigne, Appl. Phys. Lett. 65, 590 (1994).

${ }^{4}$ S. Molyneaux, H. Matsuda, A. K. Kar, B. S. Wherret, S. Okoda, and H. Nakanishi, Nonlinear Opt. 4, 299 (1993).

${ }^{5}$ D. G. Pfeiffer, T. C. Chung, D. N. Schulz, P. K. Agarwal, R. T. Garner, and M. W. Kim, J. Chem. Phys. 85, 4712 (1986).

${ }^{6}$ C. Colombi, D. Comoretto, C. Cuniberti, G. F. Musso, P. Piaggio, and G. Dellepiane, Macromol. Chem. Phys. 197, 1241 (1996).

${ }^{7}$ G. Dellepiane, C. Cuniberti, D. Comoretto, G. F. Musso, G. Figari, A. Piaggi, and A. Borghesi, Phys. Rev. B 48, 7850 (1993).

${ }^{8}$ D. Comoretto, C. Cuniberti, G. F. Musso, G. Dellepiane, F. Speroni, C. Botta, and S. Luzzati, Phys. Rev. B 49, 8059 (1994).
${ }^{9}$ Y. Shimoi, S. Abe, and X. Harigaya, Mol. Cryst. Liq. Cryst. Liq. Cryst. 267, 329 (1995).

${ }^{10}$ G. Dellepiane, C. Cuniberti, D. Comoretto, G. Lanzani, G. F. Musso, P. Piaggio, R. Tubino, A. Borghesi, C. Dell'Erba, G. Garbarino, and L. Moramarco, Phys. Rev. B 45, 6802 (1992).

${ }^{11}$ R. A. J. Janssen, L. Smilowitz, N. S. Sariciftci, and D. Moses, J. Chem. Phys. 101, 1787 (1994).

${ }^{12}$ N. S. Sariciftci, B. Kraabel, C. H. Lee, K. Pakbaz, A. J. Heeger, and D. J. Sandman, Phys. Rev. B 50, 12044 (1994).

${ }^{13}$ J. Orenstein, S. Etemad, and G. L. Baker, J. Phys. C 17, L297 (1984).

${ }^{14}$ L. Robins, J. Orenstein, and R. Superfine, Phys. Rev. Lett. 56, 1850 (1986).

${ }^{15}$ G. Dellepiane, C. Cuniberti, P. Piaggio, G. F. Musso, D. Comoretto, G. Lanzani, A. Piaggi, and A. Borghesi, Synth. Met. 51, 239 (1992).

${ }^{16}$ G. Dellepiane, C. Cuniberti, D. Comoretto, G. Lanzani, G. F. Musso, A. Piaggi, and A. Borghesi, in Frontiers of Polymers and Advanced Materials, edited by P. N. Prasad (Plenum, New York, 1994), p. 197.

${ }^{17}$ F. L. Pratt, K. S. Wong, W. Hayes, and D. Bloor, J. Phys. C 20, L41 (1987); J. Phys. D 20, 1361 (1987). 